\title{
The Southern Hemisphere VLBI Experiment program, SHEVE
}

D. L. JAUNCEY, J. E. REYNOLDS, A. K. TZIOUMIS, R. H. FERRIS, R. P. NORRIS, M. W. SINCLAIR, V. MIGENES, R. G. GOUGH and R. M. WARK Australia Telescope National Facility, CSIRO

R. A. PRESTON, D. L. MEIER, D. W. MURPHY, D. L. JONES, D. W. HOARD, MARY E. ST JOHN and L. SKJERVE

$$
\text { Jet Propulsion Laboratory }
$$

E. A. KING, P. M. MCCULLOCH, J. E. J. LOVELL and P. A. HAMILTON

$$
\text { University of Tasmania }
$$

M. E. COSTA and D. G. BLAIR

University of Western Australia

P. EDWARDS and R. W. CLAY

University of Adelaide

D. CAMPBELL-WILSON, S. W. AMY and W. B. MCADAM

University of Sydney

G. L. WHITE and P. A. JONES

University of Western Sydney, Nepean

and

G. D. NICOLSON

Hartebeesthoek Radio Astronomy Observatory

\begin{abstract}
The Southern Hemisphere VLBI Experiment (SHEVE) program is aimed at producing high-resolution images of southern radio sources. The radio telescopes of the present SHEVE array are described below and some recent results presented.
\end{abstract}

Key words: VLBI, Array

\section{Introduction}

Australia, as the continent with the major radio astronomy facilities in the Southern Hemisphere, plays an important role in very high angular resolution radio imaging. VLBI in the Southern Hemisphere is undertaken through a collaborative program involving radio telescopes at eight sites in Australia and one in South Africa, with many international connections into the US and the Asia-Pacific region. This program is known as the Southern Hemisphere VLBI Experiment, or SHEVE, and has grown out of the earlier 1982 observations (see Preston et al. 1989). More recently, a considerable effort has been undertaken to enhance both the sensitivity and $u-v$ coverage (Jauncey 1991; Preston et al. 1993).

\section{The SHEVE Telescopes}

Co-operative observing sessions of one to two weeks duration are usually scheduled three times a year through the ATNF Time Assignment Committee. Figure 1 shows a map indicating the location of the SHEVE telescopes. Participating radio telescopes include the AT LBA sites at Narrabri, Mopra and Parkes, together with 
the NASA Deep Space Network telescopes at Tidbinbilla, the Mt Pleasant Observatory near Hobart, the ESA tracking antenna near Perth and the Hartebeesthoek Radio Astronomy Observatory in South Africa. The Molonglo Observatory Synthesis Telescope, MOST, has been added for observations at $0.843 \mathrm{GHz}$ and the Alice Springs Landsat antenna has also been used occasionally with the SHEVE array. Recently, at $5 \mathrm{GHz}$ the $27.5 \mathrm{~m} \mathrm{AOTC}$ communications antenna near Perth has participated in the SHEVE program.

Observing frequencies supported by the SHEVE array include $0.843,1.7,2.3$, 4.8 and $8.4 \mathrm{GHz}$, although not all telescopes support all frequencies. MkII recording terminals are located at all sites and MkIII terminals at Parkes, Tidbinbilla, Hobart and HartRAO. The MkII data are correlated at the JPL/Caltech Block II correlator and the MkIII data at the Washington correlator. By 1994, S-2 recorders will be available with a domestic S-2 correlator.

\section{Some Recent Results}

Recent results include multi-epoch images of the nucleus of Centaurus A (NGC 5128 , PKS 1322-427). At a distance of $4 \mathrm{Mpc}(1 \mathrm{mas}=0.019 \mathrm{pc}$ ) Centaurus $\mathrm{A}$ is the nearest active galaxy to the Milky Way and the nucleus has been imaged with the SHEVE array at 2.3 and $8.4 \mathrm{GHz}$ at several epochs (Meier et al. 1989). No structural changes have been seen at $2.3 \mathrm{GHz}$ over an eight-year period although at $8.4 \mathrm{GHz}$ significant changes were seen in both flux density and core structure (Meier et al. 1993). The change in elongation of the nuclear jet over a nine-month period in 1991 indicates that it is expanding at a speed of $4.0 \pm 0.8 \mathrm{mas} / \mathrm{yr}, \mathrm{v}=0.26 \mathrm{c}$, along the $51^{\circ}$ position angle of the large-scale radio jet.

The unusually strong Einstein ring radio source PKS 1830-211 (Jauncey et al. 1991) has also been the target of multi-epoch SHEVE imaging observations (Jauncey et al. 1993) as well as with a northern global VLBI array (Jones et al. 1993). This source has varied dramatically in intensity at $8.4 \mathrm{GHz}$ and hence it is likely that an accurate estimate of the time delay in the ray paths between the two lensed images will be measurable in the future.

At $2.3 \mathrm{GHz}$ images of a number of strong southern radio galaxies and quasars have been made (Murphy et al. 1993), and a VLBI survey is under way of a sample of Parkes sources with spectral peaks in the range 0.1 to $2 \mathrm{GHz}$. Many of these sources are doubles with high brightness temperature components separated by up to $0.5^{\prime \prime}$ (King et al. 1993). The discovery of the Einstein ring radio source PKS 1830-211, which is in this sample, and the wide 6" separation found for the two high brightness-temperature components in the unusual source MSH 04-71 (Reynolds et al. 1993) raises the question: how many of such sources are gravitational lenses?

\section{References}

Jauncey, D.L., 1991, 'VLBI in Australia - A Review', A ust. J. Phys., 44, 785-803.

Jauncey, D.L., et al. 1991, 'An unusually strong Einstein ring in the radio source PKS 1830-211', Nature, $352,132-134$.

Jauncey, D.L., and the SHEVE Team, 1993, 'Southern Hemisphere Observations of PKS 1830-211', Proc. Symposium on Sub-Arcsecond Radio Astronomy, Manchester, July 1992, in press. 
Jones, D.L., and the SHEVE Team, 1993, 'Northern Hemisphere VLBI observations of PKS 1830 211', Proc. Symposium on Sub-Arcsecond Radio Astronomy, Manchester, July 1992, in press.

King, E.A., and the SHEVE Team, 1993, 'A VLBI survey of Southern Hemisphere peaked spectrum sources', Proc. Symposium on Sub-Arcsecond Radio Astronomy, Manchester, July 1992, in press.

Meier, D.L., et al. 1989, 'The high resolution structure of the Centaurus A nucleus at 2.3 and 8.4 GHz.', A atron. J., 08, $27-35$.

Meier, D.L., and the SHEVE Team, 1993, 'Multi-epoch 8.4 GHz VLBI observations of the nucleus of Centaurus A', Proc. Symposium on Sub-Arcsecond Radio Astronomy, Manchester, July 1992 , in press.

Murphy, D.W., and the SHEVE Team, 1993, '2.3 GHz VLBI images of Southern Hemisphere radio galaxies and quasars', Proc. Symposium on Sub-Arcsecond Radio Astronomy, Manchester, July 1992, in press.

Preston, R.A. et al. 1989, The Southern Hemisphere VLBI Experiment, A stron. J., 88, 1-26.

Preston, R.A., and the SHEVE Team, 1993, "The Southern Hemisphere VLBI Experiment (SHEVE)', Proc. Symposium on Sub-Arcsecond Radio Astronomy, Manchester, July 1992, in press.

Reynolds, J.E., and the SHEVE Team, 1993, 'The unusual radiosource MSH 04-71', Proc. Symposium on Sub-Arcsecond Radio Astronomy, Manchester, July 1992, in press.

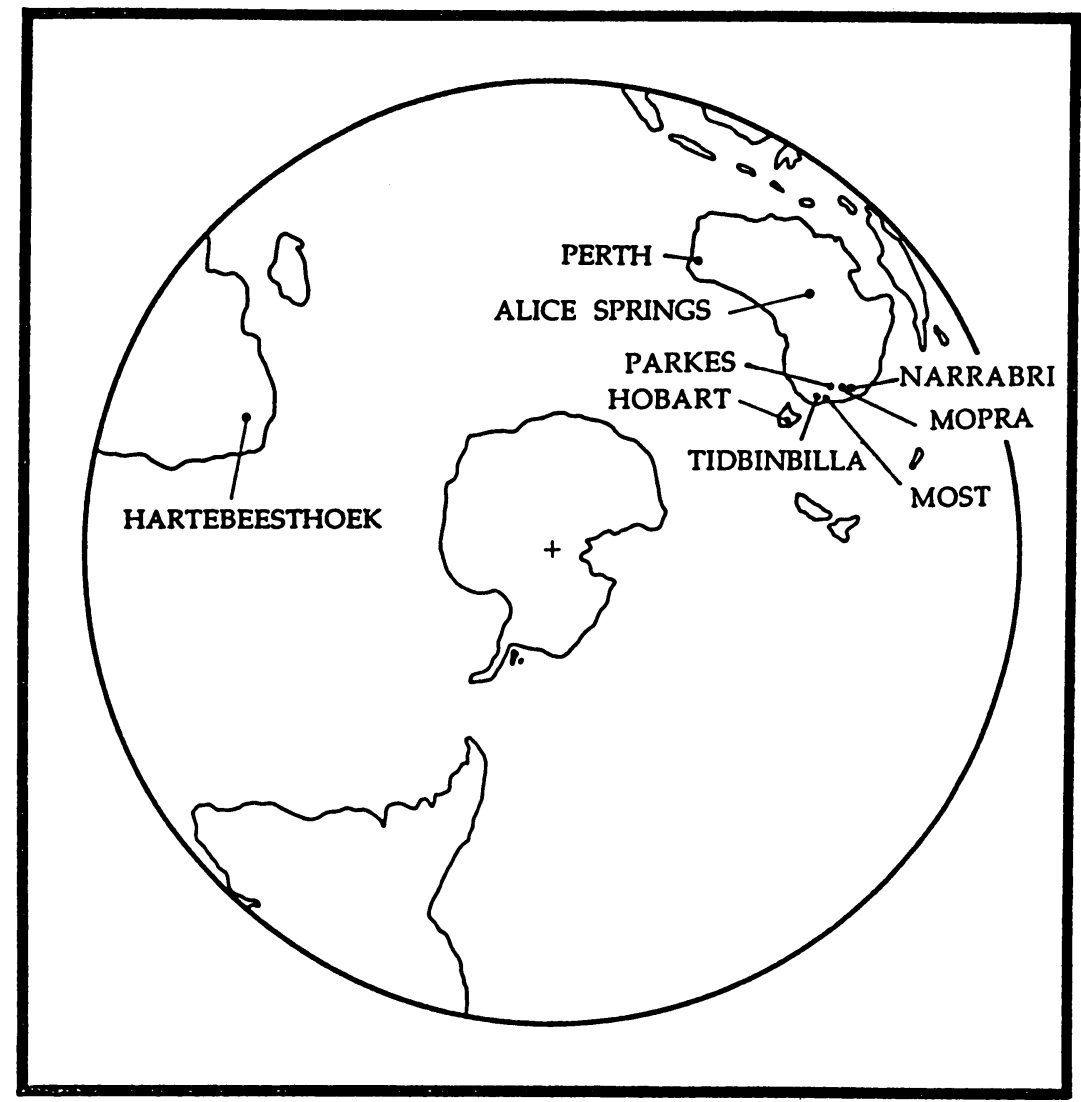

Fig. 1. The location of the telescopes of the SHEVE array. 


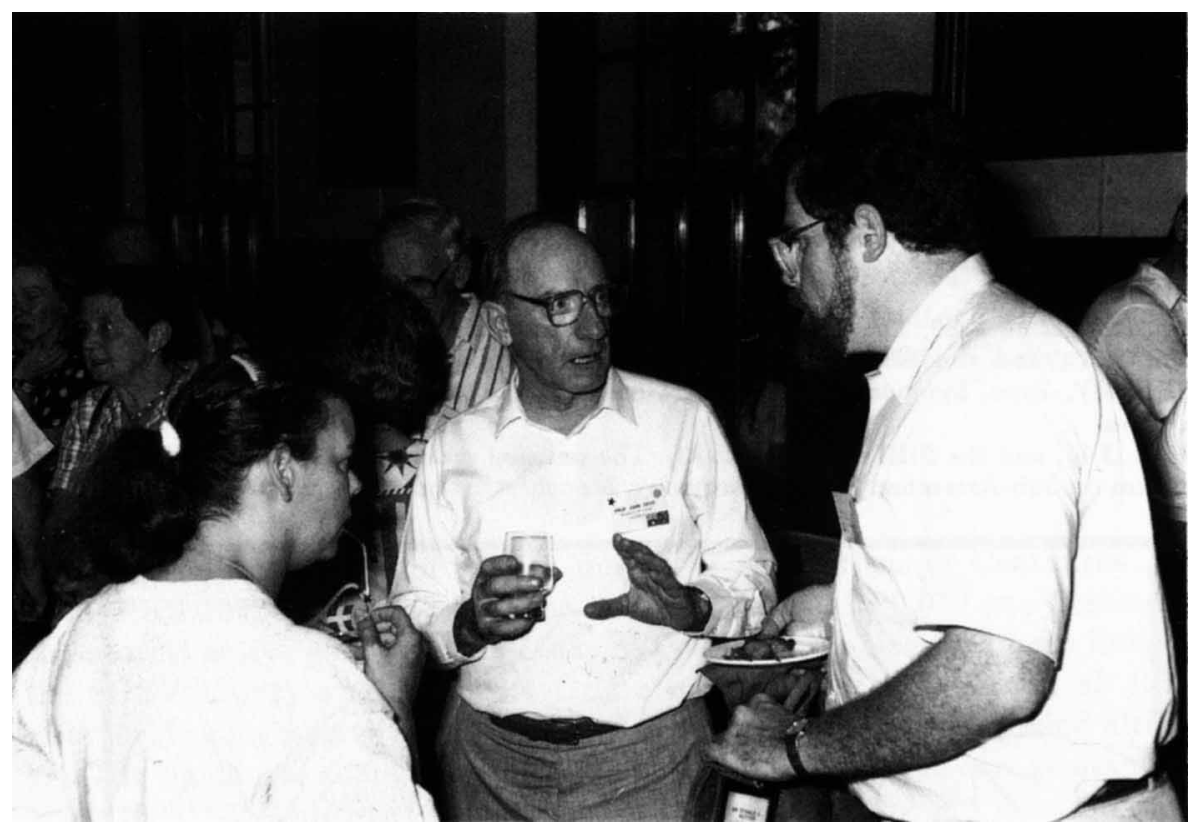

